\title{
Functionalization of yogurts with Agaricus bisporus extracts encapsulated in spray-dried maltodextrin crosslinked with citric acid
}

\author{
Cristhian R.L. Francisco ${ }^{\mathrm{a}, \mathrm{b}, \mathrm{c}}$, Sandrina A. Heleno ${ }^{\mathrm{a}, \mathrm{b}}$, Isabel P.M. Fernandes ${ }^{\mathrm{a}}$, João C.M. Barreira ${ }^{\mathrm{b}}$, \\ Ricardo C. Calhelha ${ }^{\mathrm{b}}$, Lillian Barros ${ }^{\mathrm{b}}$, Odinei Hess Gonçalves ${ }^{\mathrm{c}}$, Isabel C.F.R. Ferreira ${ }^{\mathrm{b}, *}$, \\ Maria Filomena Barreiro ${ }^{\mathrm{a}, *}$ \\ ${ }^{a}$ Laboratory of Separation and Reaction Engineering - Laboratory of Catalysis and Materials (LSRE-LCM), Polytechnic Institute of Bragança, Campus Santa Apolónia, \\ 1134, 5301-857 Bragança, Portugal \\ ${ }^{\mathbf{b}}$ Mountain Research Centre (CIMO), ESA, Polytechnic Institute of Bragança, Campus Santa Apolónia, 1172, 5301-855 Bragança, Portugal \\ c Post-Graduation Program of Food Technology (PPGTA), Federal University of Technology - Paraná - UTFPR, Campus Campo Mourão, via Rosalina Maria dos Santos, \\ 1233, CEP 87301-899, Caixa Postal: 271, Campo Mourão, PR, Brazil
}

\section{A R T I C L E I N F O}

\section{Keywords:}

Agaricus bisporus

Ultrasound extraction

Spray drying

Microencapsulation

Yogurt functionalization

\begin{abstract}
A B S T R A C T
Mushroom extracts contain bioactive compounds potentially useful to functionalize foodstuffs. Herein, alcoholic extracts of Agaricus bisporus were studied for their bioactivity and viability as functional ingredients in a food product with high water content (yogurt). Extracts were microencapsulated (to improve their stability and hydrophilicity) by spray-drying, using maltodextrin crosslinked with citric acid as encapsulating material. The effect of thermal treatment (after atomization) on crosslinking and bioactivity of microspheres was tested. The incorporation of free and thermally untreated forms resulted in yogurts with higher initial antioxidant activity (EC ${ }_{50}$ values: 214 and $272 \mathrm{mg} \cdot \mathrm{mL}^{-1}$ ) that decreased after 7 days $\left(\mathrm{EC}_{50}\right.$ values: 248 and $314 \mathrm{mg}^{\mathrm{mL}}{ }^{-1}$ ). Contrarily, thermally treated microencapsulated extracts showed higher antioxidant activity after the same period ( $\mathrm{EC}_{50}$ values, 0 days: $106 \mathrm{mg} \cdot \mathrm{mL}^{-1}$; 7 days: $48.7 \mathrm{mg} \cdot \mathrm{mL}^{-1}$ ), in result of an effective protection provided by microencapsulation with crosslinked maltodextrin and citric acid. Functionalized yogurts showed an overall maintenance of nutritional properties.
\end{abstract}

\section{Introduction}

The extensive use of synthetic additives in foods, some of them recognized as carcinogenic substances, has alerted consumers towards the need of adopting healthier habits. Moreover, their appetence for functional foods, i.e. foods promoting positive effects on health in addition to their basic nutritional purposes, is progressively increasing (Granato, Nunes, \& Barba, 2017). In fact, functional foods are integrating the daily diet of consumers and are gaining prominence worldwide. The potential global market for functional foods and beverages has been estimated to value $\$ 192$ billion by 2020 (Kaur \& Singh, 2017).

Mushrooms represent interesting sources of bioactive compounds with potential application in functional foods. They can provide immunomodulating, antitumour, anti-hypercholesterolemic, antibacterial, antifungal, anti-inflammatory, antiviral, anti-diabetic, and cardioprotective effects to consumers (Reis, Martins, Vasconcelos, Morales, \& Ferreira, 2017). Agaricus bisporus is the species with the highest consumption worldwide, showing a great potential to be used to enrich food matrices, mainly due to its high amount of ergosterol, a mycosterol with several bioactivities such as antioxidant, anti-inflammatory, antitumoral and antimicrobial, in addition to hypocholesterolemic effects (Gao et al., 2007; Gil-Ramírez, Ruiz-Rodríguez, Marín, Reglero, \& SolerRivas, 2014; Shao, Hernandez, Kramer, Rinker, \& Tsao, 2010; Yasukawa et al., 1994).

Besides selecting the bioactive ingredient (mushroom extract in the study reported herein) it is also important to choose a suitable food product suitable to be functionalized. Yogurt is among the most studied products, most likely because it is a cheap product, generally appreciated by most consumers, besides having intrinsic beneficial properties (Pereira et al., 2016; Pereira et al., 2016). Nevertheless, due to their high water content yogurts are mainly appropriate to be functionalized with hydrophilic extracts (Caleja et al., 2016; Ghorbanzade, Jafari, Akhavan, \& Hadavi, 2017; Karaaslan, Ozden, Vardin, \& Turkoglu, 2011; Karam, Gaiani, Hosri, Burgain, \& Scher, 2013; Santillán-Urquiza, Méndez-Rojas, \& Vélez-Ruiz, 2017; Singh \& Muthukumarappan, 2008). Still, the formulation of functional foods, namely dairy beverages, with

\footnotetext{
* Corresponding authors.

E-mail addresses: iferreira@ipb.pt (I.C.F.R. Ferreira), barreiro@ipb.pt (M.F. Barreiro).
} 
A. bisporus extracts was reported to increase their bioactivity, as indicated by in vitro studies that validated the antioxidant activity and cytotoxicity against tumor cells (Heleno et al., 2017). However the poor solubility of its major compound, ergosterol, both in lipophilic and hydrophilic media, may limit the application of those extracts (Barreira, Oliveira, \& Ferreira, 2014; Corrêa, Peralta, Bracht, \& Ferreira, 2017). This solubility constraint might be overcome by microencapsulating the extract with a material showing improved water compatibility such as maltodextrin, which could also allow a better stability during storage.

Among different encapsulating materials used to protect bioactive ingredients and increase their bioaccessibility, maltodextrin is a competitive solution. It was already tested with success with different bioactives such as mussel hydrolysates and several phenolic compounds (Breternitz, de V. Fidelis, Silva, Eberlin, \& Hubinger, 2017; Moser et al., 2017) and it was also studied in combination with other cross-linking molecules (e.g., cinnamyl alcohol, transglutaminase and octenyl succinic anhydride) showing the advantages of following such strategies (Khandal, Aggarwal, Suri, \& Coqueret, 2015; Nawong, Oonsivilai, Boonkerd, \& Truelstrup Hansen, 2016; Wang et al., 2014).

The application of maltodextrin itself, without any hardening or reticulation treatment, results in the ready release of the active component upon contact with the hydrophilic matrix, especially in the case of low viscosity products. Among different options, some recent works have shown the possibility to conduct maltodextrin reticulation with citric acid under certain conditions of temperature and time, resulting in materials with improved thermal and mechanical resistance (Awadhiya, Kumar, \& Verma, 2016; Castro-Cabado, Parra-ruiz, Casado, \& Román, 2016; Menzel et al., 2013). This strategy is worth investigating since it combines natural products with a low-cost process with potential implementation at industrial scale, resulting in an attractive and viable alternative to produce mycosterol-derived ingredients to functionalize food products, such as yogurts (Dias, Ferreira, \& Barreiro, 2015; Ribeiro et al., 2015). To the best of our knowledge this approach was never tested with spray-drying,

Therefore, the aim of this work was the development of a microencapsulation process to obtain microspheres containing A. bisporus extracts by spray-drying using maltodextrin cross-linked with citric acid, a novel material in the present context, as the carrier material. In order to guarantee an effective crosslinking reaction, a post thermal treatment was also studied. The obtained microcapsules were used as functionalizing agents of yogurt.

\section{Materials and methods}

\subsection{Standards and reagents}

Methanol and acetonitrile of HPLC grade, and chloroform and ethanol of analytical grade were acquired from Fisher Scientific (Lisbon, Portugal). Maltodextrin was provided by Cargill (Wayzata, MN, USA) with a dextrose equivalent of 18. Citric acid was acquired from Panreac (Barcelona, Spain). Trolox (6-hydroxy-2,5,7,8-tetra- methylchroman-2-carboxylic acid), the sterol standards (ergosterol (98\%) and cholecalciferol (98\%)), acetic acid, phosphate buffered saline (PBS), sulforhodamine B (SRB), trypan blue, trichloroacetic acid (TCA) and tris-(hydroxymethyl)aminomethane (TRIS) were purchased from Sigma (St Louis, MO, USA). Dulbecco's Modified Eagle's Medium (DMEM) and RPMI-1640 medium, fetal bovine serum (FBS), Hank's balanced salt solution (HBSS), l-glutamine, nonessential amino acid solution $(2 \mathrm{mM})$, penicillin/streptomycin solution $\left(100 \mathrm{U} \mathrm{mL}^{-1}\right.$ and $100 \mathrm{mg} \mathrm{mL}^{-1}$, respectively), and trypsin- EDTA (ethylenediaminetetraacetic acid) were from Hyclone (Logan, UT, USA). Water was treated in a Milli-Q water purification system (TGI Pure Water Systems, Greenville, SC, USA) before use. 2,2-Diphenyl-1-picrylhydrazyl (DPPH) was obtained from Alfa Aesar (Ward Hill, MA, USA). All other chemicals and solvents were of analytical grade and purchased from common suppliers.

\subsection{Obtainment and characterization of A. bisporus extract}

\subsubsection{Source material}

The samples of Agaricus bisporus L. were provided by a local mushroom producer "Mogaricus mushrooms - Sociedade Unipessoal Ltda“. They correspond to exemplars with non-conformities (e.g. small visual defects, low size etc.) to be commercialized constituting biowaste for the company. Samples were weighed, frozen, freeze-dried (Freezone 4.5 freeze dryer model 7750031, Labconco, Kansas City, MO, USA) and then reduced to powder (20 mesh).

\subsubsection{Extraction procedure}

Ultrasound-assisted extraction (UAE) was the chosen technique to obtain the extracts. It was carried out using an ultrasonic device (QSonica sonicators, model CL-334, Newtown, CT, USA), which comprise an ultrasound probe working in the range of $100-500 \mathrm{~W}$ at $20 \mathrm{kHz}$ and a digital timer. Extractions were carried out according to the procedure described by Heleno et al. (2016). Briefly, the lyophilized powdered mushroom samples ( $3 \mathrm{~g}$ ) were extracted with $100 \mathrm{~mL}$ of ethanol using previously optimized conditions ( $15 \mathrm{~min}, 375 \mathrm{w}$ ). After extraction, the extract solution was filtered through a Whatman paper filter No. 4 and the solvent evaporated under reduced pressure to obtain the Agaricus bisporus raw extract (ABRE). The obtained composition, performed according to the one reported by Heleno et al. (2016), comprised a content of $19.4 \mathrm{mg} . \mathrm{g}^{-1}$ in ergosterol.

\subsubsection{Characterization}

A. bisporus extract was characterized in what concerns antioxidant and anti-inflammatory activities and cytotoxicity. Antioxidant activity was evaluated using the DPPH radical scavenging activity assay. For this, the A. bisporus extracts were re-dissolved in ethanol at $20 \mathrm{mg} \cdot \mathrm{mL}^{-1}$. These stock solutions were successively diluted to determine $\mathrm{EC}_{50}$ values (sample concentration providing a value of $50 \%$ in the DPPH assay). DPPH radical scavenging activity was performed according to the methodology described by Heleno et al. (2012), and evaluated using an ELX800 microplate Reader (Bio-Tek Instruments, Inc., Winooski, VT, USA). The percentage of DPPH discoloration was calculated using Eq (1), where $A_{S}$ is the absorbance of the solution containing the sample at $515 \mathrm{~nm}$, and $A_{D P P H}$ is the absorbance of the DPPH solution.

DPPH scavenging activity $(\%)=100 \times \frac{A_{D P P H}-A_{S}}{A_{D P P H}}$

Anti-inflammatory activity was evaluated following the procedure reported by Taofiq et al. (2015). Briefly, the LPS-induced NO production by Murine macrophage (RAW 264.7) cell lines was determined as nitrite concentration in the culture medium. The A. bisporus extract was dissolved at $8 \mathrm{mg} \cdot \mathrm{mL}^{-1}$ in water and the nitric oxide (NO) production was determined using the Griess Reagent System kit and dexamethasone as a positive control.

For the toxicity evaluation, a primary culture cell was prepared from a porcine liver (PLP2), according to a procedure previously established by Abreu et al. (2011). The A. bisporus extract was dissolved in water at $8 \mathrm{mg} \cdot \mathrm{mL}^{-1}$ and successive dilutions were performed from the stock solution. PLP2 cells were treated for $48 \mathrm{~h}$ with the different sample solutions using sulforhodamine B assay and ellipticine was used as positive control.

\subsection{Microencapsulation of A. bisporus extract}

\subsubsection{Microencapsulation procedure}

Microencapsulation was performed by the spray-drying technique using maltodextrin crosslinked with citric acid as the encapsulating material. The used procedure and operating conditions were adapted from those described by Ribeiro et al. (2015) with some modifications. This comprised the preparation of a solution with a ABRE/maltodextrin 
ratio of $1 / 20(\mathrm{w} / \mathrm{w})$ and a citric acid content corresponding to a $\mathrm{COOH} /$ $\mathrm{OH}$ ratio (in maltodextrin basis) of 0.10 . The operating conditions were as follows: inlet temperature $170{ }^{\circ} \mathrm{C}$, outlet temperature $95^{\circ} \mathrm{C}, 90 \%$ aspirator and $20 \%$ pump $(6 \mathrm{~mL} / \mathrm{min})$. To prepare the spray solution, the ABRE $(1 \mathrm{~g})$ was first dissolved in ethanol $(10 \mathrm{~mL})$ followed by the addition of water $(90 \mathrm{~mL})$ to obtain a homogeneous solution. Thereafter citric acid ( $3.5 \mathrm{~g}$ ) was added under stirring until complete dissolution, followed by the addition of Tween 20 (T20, $20 \mu \mathrm{L}$ ). Finally, maltodextrin $(20 \mathrm{~g})$ was added while maintaining stirring until complete homogenization of the solution. The final produced solution has a proximate solids content of $20 \%(\mathrm{w} / \mathrm{w})$. This procedure was performed immediately prior to the solution atomization. The equipment used was a Mini Spray Dryer B-290 (Buchi, Flawil, Switzerland) equipped with an Inert Loop B-295 to promote closed circuit circulation under nitrogen atmosphere. The nozzle used had a diameter of $0.7 \mathrm{~mm}$ and the atomized volume was $100 \mathrm{~mL}$. Microspheres containing maltodextrin crosslinked with citric acid, but without the extract, were also produced to be used as control. After atomization, the produced microspheres with $A$. bisporus extract and the control microspheres (without extract) were maintained in an oven at $140{ }^{\circ} \mathrm{C}$ for $4 \mathrm{~h}$, according to the procedure described in the work of Castro-Cabado et al. (2016) to further extend the crosslinking reaction. The following samples were obtained: (i) thermally untreated microspheres containing A. bisporus extract (UME), (ii) thermally treated microspheres containing A. bisporus extract (TME); (iii) thermally untreated control microspheres (UCM) and (iv) thermally treated control microspheres (TCM).

\subsubsection{Microencapsulation yield, efficiency and load}

The microencapsulation process was characterized in terms of yield and encapsulation efficiency. Yield, an estimative of the material losses during the process, was determined gravimetrically as the ratio between the weight of the recovered microspheres and the weight of the atomized materials. Encapsulation efficiency, expressed in terms of ergosterol (major compound identified in the extract), was determined by HPLC-UV using the ratio between the experimentally quantified encapsulated extract and the theoretical one. The equipment consisted of an integrated system with a pump (Knauer, Smartline system 1000, Berlin, Germany), a degasser system (Smartline manager 5000), an auto-sampler (AS-2057 Jasco, Easton, MD) and a UV detector (Knauer Smartline 2500). For that, ergosterol was quantified based on a calibration curve obtained from a commercial standard using the internal standard method with cholecalciferol as the internal standard (Barreira et al., 2014) and data analyzed using Clarity 2.4 Software (DataApex). Microcapsules load was thereafter determined as the ratio $(w / w)$ between the effectively microencapsulated extract and the obtained microspheres amount.

\subsubsection{Characterization of the obtained microspheres}

Microspheres were characterized in terms of size, by thermogravimetric analysis (TGA) and by Fourier Transform Infrared Spectroscopy (FTIR). Additionally, antioxidant and anti-inflammatory activities and toxicity were evaluated following the methodologies described in Section 2.2.3. with minor modifications.

The size distribution was analyzed on a Malvern Mastersizer 3000 equipped with a Hydro MV dispersion unit (Malvern, UK) using ethanol as the solvent. Data was acquired and treated with Mastersizer software version 3.10. Both size distributions, in number and volume, were determined and respective averages calculated.

TGA was performed on a NETZSCH model TG 209 F3 Tarsus (Selb, Germany) under the conditions described by Castro-Cabado et al. (2016). Briefly, the base materials (used as reference) and the obtained microspheres were heated in aluminum crucibles from 10 to $600 \mathrm{C}$ at a heating rate of $10^{\circ} \mathrm{C} \cdot \mathrm{min}^{-1}$ under nitrogen flow $\left(20 \mathrm{~mL} \cdot \mathrm{min}^{-1}\right)$. Thermograms were obtained and treated with Netzsch Proteus Thermal Analysis software, version 5.2.1.

FTIR spectra were obtained on ABB Inc. FTIR, model MB3000
(Quebec, Canada) in transmittance mode by using $\mathrm{KBr}$ pellets with a sample concentration of $1 \%(\mathrm{w} / \mathrm{w})$. Spectra were recorded between 650 and $4000 \mathrm{~cm}^{-1}$ at a resolution of $16 \mathrm{~cm}^{-1}$ and cumulative 32 scans. Spectra were acquired and treated (baseline correction) using the software Horizon MB v.3.4. Due to experimental difficulties associated with the preparation of the $\mathrm{KBr}$ pellets, ABRE and citric acid analysis were performed using a Miracle single reflection horizontal ATR accessory from Pike Technologies (Madison, WI, USA) using the same conditions.

\subsection{Yogurts added with $A$. bisporus extract}

\subsubsection{Samples preparation}

The base yogurt was purchase from a local supermarket. The nutritional composition according to the label reference, per $100 \mathrm{~g}$, was as follows: energy content of $75 \mathrm{kcal} ; 3.8 \mathrm{~g}$ of lipids corresponding to $2.4 \mathrm{~g}$ of saturated fatty acids; $4.7 \mathrm{~g}$ of carbohydrates corresponding to sugars; $5.0 \mathrm{~g}$ of proteins and $0.15 \mathrm{~g}$ of salt.

Four sample groups, each one comprising $50 \mathrm{~g}$ of yogurt, were prepared: a negative control (NCY) corresponding to the base yogurt; a positive control (PCY) corresponding to yogurt incorporated with free A. bisporus extract $(40 \mathrm{mg} / 50 \mathrm{~g})$; a yogurt containing the $A$. bisporus thermally untreated microspheres $(2.5 \mathrm{~g} / 50 \mathrm{~g}$ ) (YUME); and a yogurt containing the $A$. bisporus thermally treated microspheres $(2.5 \mathrm{~g} / 50 \mathrm{~g})$ (YTME). The used microsphere's amount was selected in order to incorporate an amount of extract equivalent to the one used in the PCY sample.

\subsubsection{Evaluation of bioactive and nutritional properties}

All samples were evaluated for their nutritional composition, antioxidant and anti-inflammatory activities and cytotoxicity, immediately after preparation (t0) and after a period of seven days under storage at $4{ }^{\circ} \mathrm{C}(\mathrm{t} 7)$. Antioxidant and anti-inflammatory activities and cytotoxicity were evaluated using the methodologies previously described in Section 2.2.3 with some modifications.

Nutritional composition (moisture, protein, fat, carbohydrates and ash) was evaluated using the AOAC procedures (AOAC, 2016). Namely, protein content (Nx6.38) was determined using the Kjeldahl method; fat content by Soxhlet extraction with petroleum ether and ash content was by incineration at $600 \pm 15^{\circ} \mathrm{C}$. Total carbohydrates were calculated by difference. Energy was calculated according to Eq. (2), where PW, CW and LW are, respectively, the weight of proteins, carbohydrates and lipids in $\mathrm{g}$.

Energy $(\mathrm{kcal})=4 \times(\mathrm{PW}+\mathrm{CW})+9 \times \mathrm{LW}$

Fatty acids were determined by gas chromatography Dani 1000 (Cologno Monzese, MI, Italy) coupled with a flame ionization detector (GC-FID) according to the procedure described by Reis et al. (2011), by analyzing the petroleum ether extract previously obtained for the fat determination. Identification was made by comparison of the relative retention times of fatty acid methyl esters with standards. Results were expressed as relative percentages.

Free sugars were determined by HPLC with refractive index (IR) detection (Knauer Smartiline 2300) using a Eurospher 100-5 $\mathrm{NH}_{2}$ column $\left(5 \mu \mathrm{m}, 250 \times 4.6 \mathrm{~mm}\right.$, Knauer) operating at $35^{\circ} \mathrm{C}$. The mobile phase was acetonitrile/deionized water, 70:30 (v/v) at a flow rate of $1 \mathrm{~mL} . \mathrm{min}^{-1}$ (Heleno, Barros, Sousa, Martins, \& Ferreira, 2009). Sugars were identified by comparison with standard's retention times. Quantification was performed by the internal standard method (melezitose) and data analyzed using the software Clarity 2.4 (DataApex).

\subsection{Statistical analysis}

All statistical tests were performed at a 5\% significance level using IBM SPSS Statistics for Windows, version 22.0. (IBM Corp., Armonk, NY, USA). Results were expressed as mean \pm standard deviation, 
maintaining the significant numbers allowed by the magnitude of the standard deviation.

Bioactivity of the studied samples was compared by means of an analysis of variance (ANOVA), followed by Tukey's test (homoscedastic distributions) or Tamhane's T2 test (heteroscedastic distributions). The fulfillment of the one-way ANOVA requirements, specifically the normal distribution of the residuals and the homogeneity of variance, was tested by means of the Shapiro Wilk's and the Levene's tests, respectively. Furthermore, an ANOVA with type III sums of squares was performed using the general linear model (GLM) procedure, to compare the parameters evaluated in the prepared yogurts. The dependent variables were analyzed using a 2-way ANOVA with the factors "functionalized yogurt" (FY) and "storage time" (ST). When a statistically significant interaction was detected among these two factors, they were evaluated simultaneously by the estimated marginal mean plots for all levels of each factor. On the contrary, if no statistical significant interaction was found, means were compared using Tukey's multiple comparison test, after checking the equality of variances through a Levene's test. A $t$-student test was applied in the case ST, since there were less than three groups.

In addition, a Linear Discriminant Analysis (LDA) was used to compare the effect of FY over the assayed parameters. A stepwise technique was applied, considering the Wilks' $\Lambda$ test with the usual probabilities of F (3.84 to enter and 2.71 to be removed) for variable selection. The matrix of results was composed by $72 \times 21$ values $(72$ rows corresponding to the analyzed samples and 21 columns corresponding to the evaluated parameters). The relationship between single categorical dependent variables (functionalized yogurts) and the quantitative independent variables (results obtained in the laboratorial assays) was estimated. To verify the significance of the canonical discriminating functions, Wilk's $\Lambda$ test was used. A leaving-one-out cross validation procedure was carried out to assess the model performance.

\section{Results and discussion}

\subsection{Microencapsulation of A. bisporus}

\subsubsection{Yield, efficiency load and size determination}

The microencapsulation of $A$. bisporus extract by the spray-drying technique using maltodextrin crosslinked with citric acid, under the used scale (atomization of $100 \mathrm{ml}$ ) presented yield of $35 \%$ and encapsulation efficiency of $40.2 \%$ (determined by HPLC in terms of ergosterol). Microspheres load, which represents the extract content per $\mathrm{g}$ of microspheres was $16.4 \mathrm{mg} / \mathrm{g}$. This parameter is important since it provides the content of the active principle in the final material allowing to determine the microspheres amount needed to functionalize a certain food matrix. Based on the achieved value $(16.4 \mathrm{mg} / \mathrm{g})$ an amount of $2.5 \mathrm{~g}$ of microspheres was estimated as equivalent to $40 \mathrm{mg}$ of free extract justifying the amounts used in the yogurt samples preparation (see Section 2.4.1. Samples preparation).

Size distributions were primarily determined for UME sample and further verified for TME. Fig. 1A and B represent a comparison of their size distributions in volume and number, respectively. Thus, microspheres had a size distribution in volume ranging from 2.3 to $25.30 \mu \mathrm{m}$. The corresponding average sizes for UME and TME were, respectively, 11.34 and $13.42 \mu \mathrm{m}$. The distribution in number varied from 0.50 to $1.68 \mu \mathrm{m}$ leading to average sizes of 1.03 and $0.85 \mu \mathrm{m}$, respectively for UME and TME samples. In conclusion, the thermal treatment has a minimal impact on the particle size distribution, namely a slight increase on the average size in volume and a slight decrease on the average size in number, which might be due to the occurrence of particles agglomeration during the thermal treatment.

\subsubsection{TG/DTG and FTIR analysis}

The obtained microspheres, as well as the used starting materials, were characterized by TG/DTG. The purpose was to assess the thermal stability of the base materials and check if the conditions used in the atomization process were, by itself, sufficient to ensure the crosslinking between maltodextrin and citric acid, as well as the advantages of carrying out the additional step of thermal treatment after the atomization process. For that, the base materials (maltodextrin, citric acid, ABRE and T20) were analyzed individually, as well as the control microcapsules (UCM and TCM) and the microspheres containing the A. bisporus extract (UME and TME).

Analyzing the base materials (Fig. S1, Supplementary Material), maltodextrin, citric acid and T20 presented degradation profiles with starting degradation temperatures above $170{ }^{\circ} \mathrm{C}\left(200,187\right.$ and $261^{\circ} \mathrm{C}$, respectively), meaning that they were resistant to both the used spraydrying and thermal post-treatment conditions $\left(170\right.$ and $140{ }^{\circ} \mathrm{C}$, respectively). In what concerns $\mathrm{ABRE}$, a degradation profile in the range $130-375^{\circ} \mathrm{C}$ was observed, with a fraction of around $3 \%$ degrading before $170^{\circ} \mathrm{C}$. However, since the spray-drying process is almost instantaneous and the degradation of ergosterol (the major component in the extract) starts around $183^{\circ} \mathrm{C}$, the impact of the used atomization temperature $\left(170^{\circ} \mathrm{C}\right)$ on the extract properties, namely on bioactivity may not be relevant.

Fig. 2 presents the thermal degradation profiles of the control microspheres (UCM and TMC) and of the ones containing the extract (UME and TME). Comparatively with pure maltodextrin, they have shown a slower degradation pattern, which is extended to higher temperatures, evidencing that both samples (with and without treatment) have fractions in their composition thermally more stable than maltodextrin itself. The same was observed by Stone, Gosavi, Athauda, and Ozer (2013), where nanofibers of alginate/polyvinyl alcohol treated with citric acid presented a degradation profile slower than that of the base nanofibers. Also, Awadhiya et al. (2016) found a similar behavior for agarose films crosslinked with citric acid. Additionally, as in the present study, maltodextrin crosslinked with citric acid resulted in a final residue fraction higher than the one obtained for the former material (maltodextrin, 2.49\%), fact associated with an effective crosslinking of the material. When comparing the effect of thermal treatment on samples without extract, the one without thermal treatment after atomization (UCM) has shown a slight slower degradation profile reflected in a slight higher final residue (18.47\%, comparatively with $16.09 \%$ for TCM). This trend was much more noticeable for the samples incorporating the extract $(17.76 \%$, for UME, comparatively with $6,31 \%$ for TME). This fact may not be supported by the occurrence of ABRE degradation (estimated in 3\%), but also on the establishment of different molecular interactions among the microsphere's components. In fact, the thermal degradation is quite similar, until $400{ }^{\circ} \mathrm{C}$, between samples of the same series (both thermally untreated samples showed a residue at $400{ }^{\circ} \mathrm{C}$ around $25 \%$, whereas the treated ones showed a value of $20 \%$ ). Moreover, one may observe that the weight lost associated with the fourth assigned degradation region (273-426 ${ }^{\circ} \mathrm{C}$; see Table S1 in Supplemental Material) increased for the samples containing the extract (around $50 \%$ of the weight lost is associated with this degradation region, whereas for the samples without extract $50 \%$ of the weight lost is concentrated on the third degradation zone $\left(196-320^{\circ} \mathrm{C}\right)$. This behavior is compatible with the observance of a thermally more resistant material due to the presence of extract, nevertheless the facilitated thermal degradation of the sample TME for temperatures higher than $400{ }^{\circ} \mathrm{C}$.

Fig. 3 presents the FTIR spectra of the base matrix materials (maltodextrin and citric acid) together with the produced microspheres, before and after thermal treatment (UCM and TCM, respectively). Citric acid crosslinking with maltodextrin through esterification is evidenced by the disappearance of the two sharp peaks previously appearing in the citric acid spectrum (1748 and $1693 \mathrm{~cm}^{-1}$ ) and the appearance of a new peak in the carbonyl region $\left(1732 \mathrm{~cm}^{-1}\right)$ assigned to the ester carbonyl stretching, both in UCM and TCM spectra. Also in this region, a peak assigned at $1646 \mathrm{~cm}^{-1}$ is present in UCM and TCM samples from the stretching of carbonyls present in the maltodextrin. Comparing the 

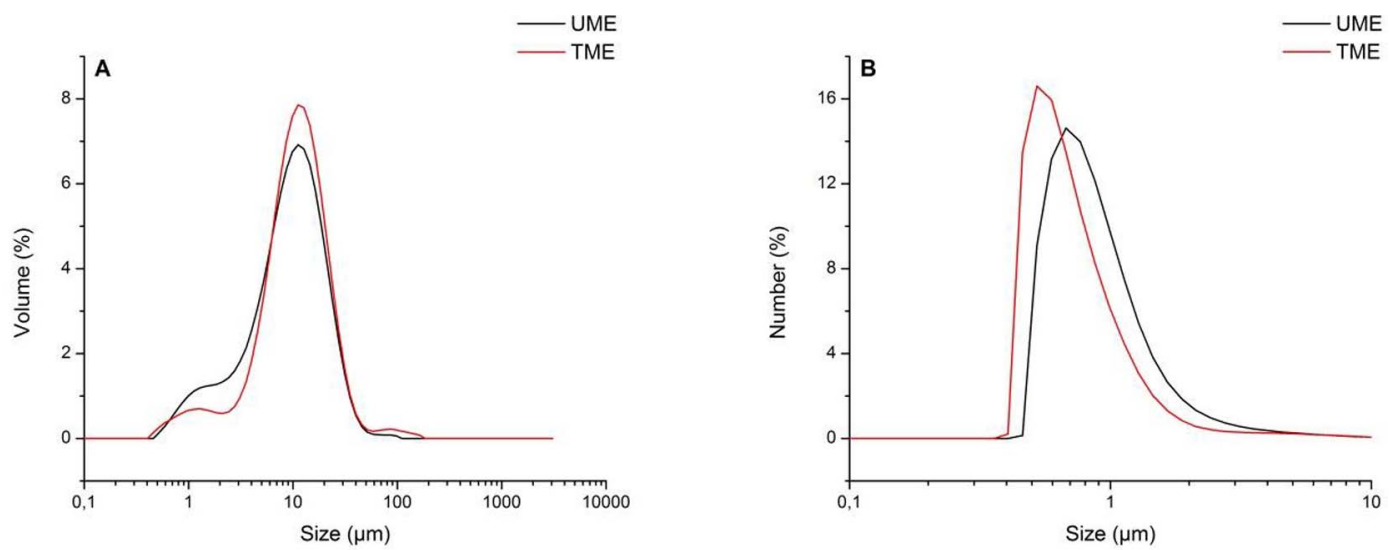

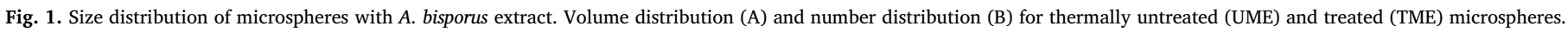

spectra of UCM and TCM, and in reference to the $2932 \mathrm{~cm}^{-1}$ vibration (invariant vibration ascribed to the $\mathrm{C}-\mathrm{H}$ stretching region), an intensification of the $1732 \mathrm{~cm}^{-1}$ peak occurs in the TCM spectra, fact associated with the extension of the crosslinking reaction due to the applied thermal treatment. The same trend was observed for the samples incorporating the extract (UME and TME). Additionally, when comparing TCM with TME an intensification of the peak at $1732 \mathrm{~cm}^{-1}$ is also observed in this last case, fact that can be justified by the citric acid reaction with the extract itself (Fig. S2. Supplementary Material).

\subsection{Bioactive properties of A. bisporus extract and produced microcapsules}

The obtained $A$. bisporus extract and produced microspheres (UCM,
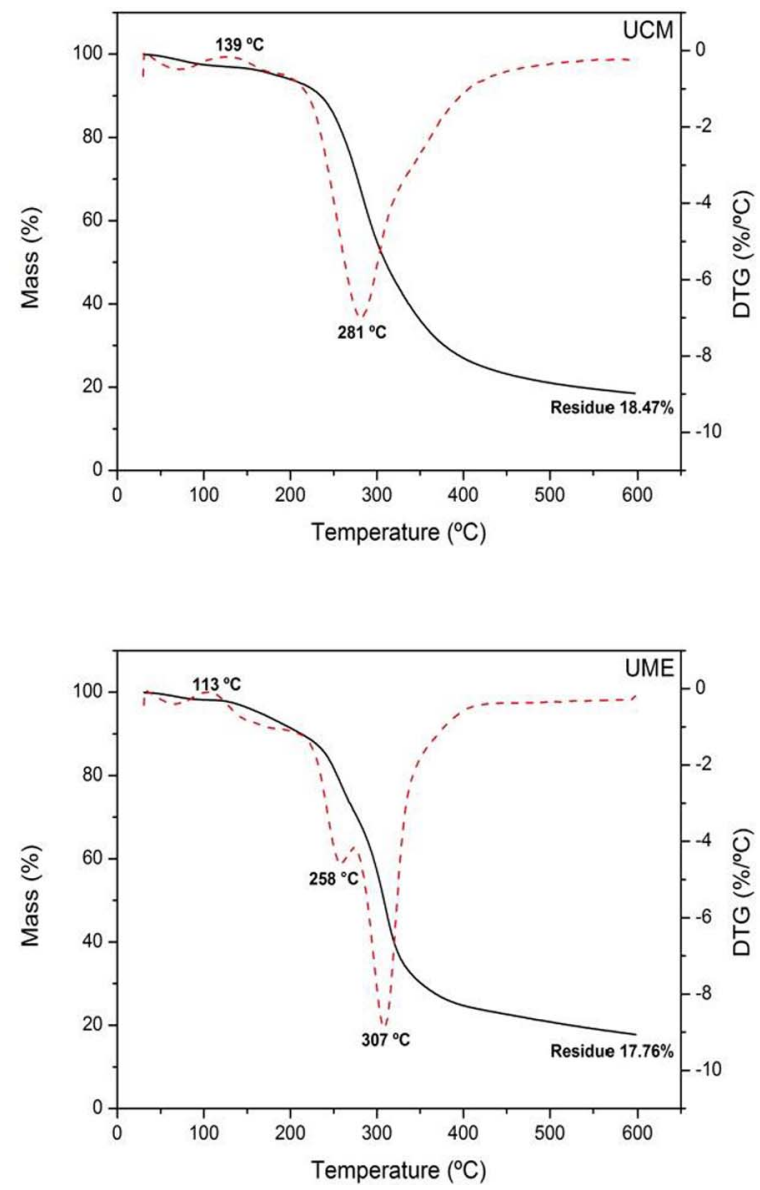

TCM, UME and TME) were evaluated for their radical scavenging capacity, cytotoxicity and anti-inflammatory activity (Table 1). As expected, $\mathrm{ABRE}$ presented the highest scavenging activity $\left(\mathrm{EC}_{50}\right.$ value of $4.3 \mathrm{mg} \cdot \mathrm{mL}^{-1}$ ), followed by the TME sample with an average $\mathrm{EC}_{50}$ value of $13.0 \mathrm{mg} \cdot \mathrm{mL}^{-1}$. The control microspheres (UCM and TCM) showed very low scavenging activity, especially the untreated ones (UCM), which did not reach $50 \%$ of activity up to the maximum of the assayed concentration ( $400 \mathrm{mg} \cdot \mathrm{mL}^{-1}$ ). In the cytotoxicity evaluation, neither one exerted an acute inhibitory effect (up to the maximum assayed concentration of $400 \mu \mathrm{g} \cdot \mathrm{mL}^{-1}$ ). Concerning anti-inflammatory activity, ABRE gave an $\mathrm{IC}_{50}$ value of $250 \mu \mathrm{g} . \mathrm{mL}^{-1}$ while the other tested samples (TCM, UCM, TME and UME) showed no activity.
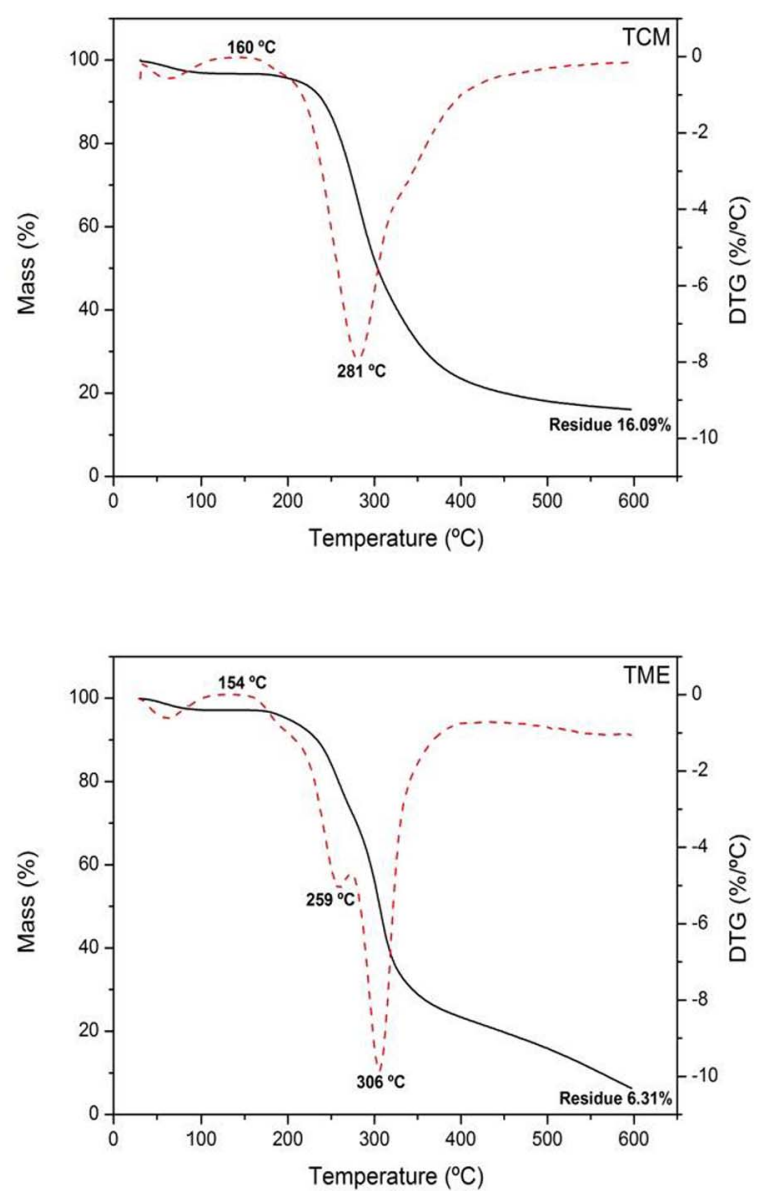

Fig. 2. Degradation profile of control samples (UCM and TCM) and microspheres with A. bisporus extract (UME and TME). 




Fig. 3. FTIR spectra of base matrix materials (maltodextrin: MD and citric acid: CA) and obtained microspheres without (UCM) and with (TCM) thermal treatment. 
Table 1

Bioactive properties of Agaricus bisporus raw extract (ABRE), thermally treated microspheres with extract (TME), thermally untreated microspheres with extract (UME), thermally treated control microspheres (TCM) and thermally untreated control microspheres (UCM). Values are indicated as mean \pm standard deviation.

\begin{tabular}{|c|c|c|c|c|}
\hline & & $\begin{array}{l}\text { Antioxidant } \\
\text { activity }\left(\mathrm{EC}_{50} \text {, }\right. \\
\mathrm{mg} / \mathrm{mL})\end{array}$ & $\begin{array}{l}\text { Cytotoxicity } \\
\left(\mathrm{GI}_{50}, \mu \mathrm{g} / \mathrm{mL}\right)\end{array}$ & $\begin{array}{l}\text { Anti- } \\
\text { inflammatory } \\
\text { activity (IC }{ }_{50} \text {, } \\
\mu \mathrm{g} / \mathrm{mL})\end{array}$ \\
\hline & & $\begin{array}{l}\text { DPPH } \\
\text { scavenging } \\
\text { activity }^{1}\end{array}$ & $\begin{array}{l}\text { PLP2 cell } \\
\text { line }^{2}\end{array}$ & NO inhibition ${ }^{3}$ \\
\hline ABRE & & $4.3 \pm 0.1 \mathrm{~d}$ & $>400$ & $250 \pm 12$ \\
\hline TME & & $13.0 \pm 0.2 \mathrm{c}$ & $>400$ & $>400$ \\
\hline UME & & $64 \pm 1 b$ & $>400$ & $>400$ \\
\hline TCM & & $234 \pm 3 a$ & $>400$ & $>400$ \\
\hline UCM & & $>400$ & $>400$ & $>400$ \\
\hline$p$-value & Homoscedasticity $^{4}$ & $<0.001$ & - & - \\
\hline$(n=18)$ & 1-way ANOVA ${ }^{5}$ & $<0.001$ & - & - \\
\hline
\end{tabular}

${ }^{1} \mathrm{EC}_{50}$ for Trolox (positive control): $42 \mu \mathrm{g} / \mathrm{mL}^{2}{ }^{2} \mathrm{GI}_{50}$ for ellipticine (positive control): $3 \mu \mathrm{g} /$ $\mathrm{mL} .{ }^{3} \mathrm{IC}_{50}$ for dexamethasone (positive control): $16 \mu \mathrm{g} / \mathrm{mL} .{ }^{4}$ Homoscedasticity was tested by Levene test: homoscedasticity, $p>.05$; heteroscedasticity, $p<.050 .{ }^{5} p<.050$ indicates that the mean value of at least one component is significantly different.

activity along with storage time while PCY and YUME showed an opposite behavior. Summarizing, results suggest that PCY and YUME samples, which present a higher initial antioxidant activity, suffer a decrease after the 7 days under storage, which may be associated with ABRE degradation. In the PCY case, it was motivated by the direct contact of the extract with the food matrix, whereas in the YUME case, possibility due to a prompt release of the extract to the food matrix and subsequent degradation. In opposition, YTME resulted in higher antioxidant activity, as already observed for the isolated microspheres. Additionally, after 7 days of storage antioxidant activity increased, fact associated with a more effective protection of the extract when thermal treatment was used. This may be explained by the effective crosslinking of the material when spray drying was used coupled to thermal treatment. This may be related to the effective reaction of citric acid both with maltodextrin and, at minor extent, with ABRE which is in agreement with TGA and FTIR, resulting in substances with improved antioxidant activity.

\subsection{Nutritional properties of the functionalized yogurts}

Nutritional properties were evaluated as a function of the used functionalization type (FY: NCY, PCY, YUME and YTME) and storage time (ST: 0 and 7 days). Additionally, the interaction between the two factors (FY and ST) was also analyzed and the changes induced by FY and ST were only classified in cases where the interaction $(\mathrm{FY} \times \mathrm{ST})$ was not significant $(\mathrm{p}>.050$ ). If significant, the corresponding parameters were characterized by analyzing the corresponding Estimated Marginal Means (EMM) plots (data not shown) obtained in the GLM procedure.

For nutritional composition (Table 2), the interaction among factors was significant for all the examined cases, with the exception of lactose $(\mathrm{p}=.574)$, limiting the classification of results according to FY or ST to that single parameter. On the other hand, ST induced significant changes only on carbohydrates, which tended to increase along time, while at least one FY showed a statistically different valued in comparison with the remaining FY, fact valid for all nutritional parameters. Only lactose results were classified, which have clearly shown that yogurts functionalized with microspheres had lower lactose contents. Still, from the EMM plots one may conclude that NCY contain the highest levels of fat, ash and galactose, while YTME presented the lowest moisture content (together with YUME), fat, protein and energy content. No unequivocal trend can be pointed out in the case of carbohydrate contents, either concerning FY effect, as well as ST. The nutritional profiles were generally in agreement with those reported previously for yogurt samples (Caleja et al., 2016).

Besides the relevance of nutritional composition, it is also important to evaluate the fatty acids profile, as their maintenance throughout storage is taken as a suitable indicator of preservation (Barreira, Pereira, Oliveira, \& Ferreira, 2010; (Pereira et al., 2016; Pereira et al., 2016). As indicated in Table 3 , the interaction between FY and ST was significant in most cases, except for C18:1n9c, SFA and MUFA. NCY presented the highest values of C18:1n9c and MUFA, and the lowest contents in SFA. Among the fatty acids that could not be classified due to the statistically significant interaction $\mathrm{FY} \times \mathrm{ST}$, EMM showed a tendency pointing out higher contents of C12:0 and C14:0 in YUME samples, while C16:0 tended to lower contents in NCY. In what concerns to ST effects, C18:1n9c and C18:2n6c tended towards lower values after 7 days, in contrast with SFA, which was higher in stored yogurts. Besides the former fatty acids, ST had a significant effect only in C16:0 and PUFA, but no overall tendencies were observable in these two cases.

In general, these results might be understood as offering new possibilities for the dairy product market, namely by using the developed yogurts as effective vehicles for bioactive ingredients, which otherwise would not be suitable to be incorporated into such type of food matrix.

Nevertheless, and despite the overall maintenance of the studied characteristics, the expansion of the developed yogurts into new consumer markets would also require the standardization of their sensory characteristics, in order to achieve a satisfying consumer acceptance (Gaze et al., 2015). Therefore, it will be mandatory to optimize formulations from a sensory point of view, with high potential success in consumer marketing. Further research should evaluate pilot-plant prototypes and confirm drivers of liking with larger groups of consumers (Janiaski, Pimentel, Cruz, \& Prudencio, 2016).

\subsection{Linear discriminant analysis}

After analyzing individual differences among each FY or ST, it became clear that they are mostly related with FY. Therefore, it is of interest knowing which of those differences are more related to each FY type. This can be done by comparing all parameters simultaneously by Linear Discriminant Analysis (LDA). This statistical tool allows identifying the compositional changes with highest relevance for a determined factor level (represented in this case by each FY), ultimately verifying their discriminant ability. Accordingly, LDA was performed to evaluate the correlations among FY (entered as categorical dependent variables) and the data matrix of results (quantitative independent variables). The significant independent variables were selected through the stepwise method, according to Wilks' $\lambda$ test. Only variables with a statistically significant classification performance $(p<.050)$ were maintained by the statistical model.

The three defined discriminant functions included $100.0 \%$ (first function: $94.5 \%$; second function: $4.3 \%$; third function: $1.2 \%$ ) of the observed variance (Fig. S4, Supplementary Material). Among the 19 variables included in the analysis, the model selected water, galactose, carbohydrates, energy, C6:0, C16:0, C18:1n9 and DPPH scavenging activity as the ones with significant discriminant effect, meaning that these were the variables with highest changes among each prepared FY.

In terms of spatial distribution, function 1 separated primarily YTME (negative end) and NCY (positive end), particularly due to its correlation with DPPH scavenging activity, which presented minimum $\mathrm{EC}_{50}$ values in YTME and maximum $\mathrm{EC}_{50}$ values in NCY (a value of $400 \mathrm{mg} \cdot \mathrm{mL}^{-1}$ was used in this case, since the $\mathrm{EC}_{50}$ could not be calculated). Function 2, in turn, was more highly correlated with water and energy contents, placing the markers corresponding to PCY and YUME in remote positions, mainly due to their differences and water content and energy (both higher in PCY). On the other hand, markers corresponding to PCY and YUME were similarly distributed through 
Table 2

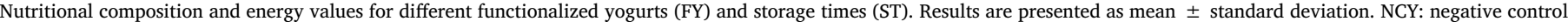

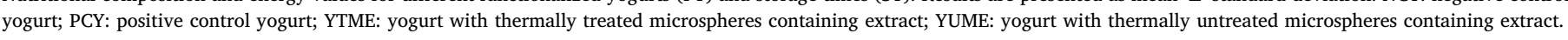

\begin{tabular}{|c|c|c|c|c|c|c|c|c|c|}
\hline & & Moisture & Fat & Protein & Ash & Carbohydrates & Galactose & Lactose & Energy \\
\hline \multirow[t]{5}{*}{ FY } & NCY & $85 \pm 1$ & $3.5 \pm 0.1$ & $4.6 \pm 0.2$ & $0.84 \pm 0.04$ & $5.8 \pm 0.2$ & $0.93 \pm 0.05$ & $4.5 \pm 0.2 \mathrm{a}$ & $73 \pm 1$ \\
\hline & PCY & $86 \pm 1$ & $3.4 \pm 0.2$ & $4.7 \pm 0.2$ & $0.74 \pm 0.05$ & $5.9 \pm 0.2$ & $0.82 \pm 0.05$ & $4.6 \pm 0.2 \mathrm{a}$ & $73 \pm 2$ \\
\hline & YTME & $82 \pm 1$ & $2.3 \pm 0.2$ & $4.5 \pm 0.2$ & $0.76 \pm 0.05$ & $6.2 \pm 0.5$ & $0.79 \pm 0.05$ & $4.1 \pm 0.2 b$ & $64 \pm 1$ \\
\hline & YUME & $82 \pm 1$ & $3.0 \pm 0.2$ & $4.6 \pm 0.1$ & $0.79 \pm 0.05$ & $5.7 \pm 0.5$ & $0.86 \pm 0.05$ & $3.9 \pm 0.2 c$ & $69 \pm 2$ \\
\hline & ANOVA $p$-value $(\mathrm{n}=18)^{2}$ & $<0.001$ & $<0.001$ & 0.033 & $<0.001$ & $<0.001$ & $<0.001$ & $<0.001$ & $<0.001$ \\
\hline \multirow[t]{3}{*}{ ST } & 0 days & $84 \pm 2$ & $3.2 \pm 0.4$ & $4.6 \pm 0.2$ & $0.79 \pm 0.05$ & $5.7 \pm 0.3$ & $0.85 \pm 0.05$ & $4.3 \pm 0.3$ & $70 \pm 5$ \\
\hline & 7 days & $84 \pm 2$ & $2.9 \pm 0.5$ & $4.6 \pm 0.1$ & $0.78 \pm 0.05$ & $6.2 \pm 0.4$ & $0.85 \pm 0.05$ & $4.3 \pm 0.3$ & $70 \pm 4$ \\
\hline & ANOVA $p$-value $(\mathrm{n}=36)^{3}$ & 0.839 & 0.051 & 0.176 & 0.355 & $<0.001$ & 0.729 & 0.881 & 0.982 \\
\hline $\mathrm{FY} \times \mathrm{ST}$ & $p$-value $(\mathrm{n}=72)^{4}$ & 0.036 & 0.012 & 0.027 & 0.001 & $<0.001$ & $<0.001$ & 0.574 & $<0.001$ \\
\hline
\end{tabular}

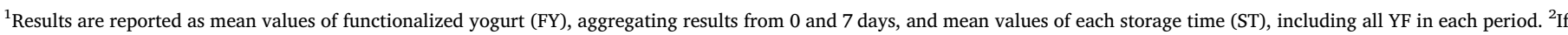

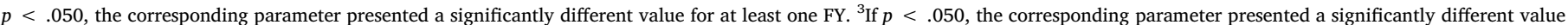
among both ST. ${ }^{4}$ If $p<.050$, the interaction among factors is significant; in this case, the multiple comparisons could not be classified.

Table 3

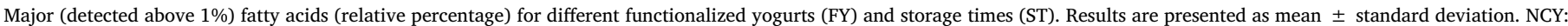

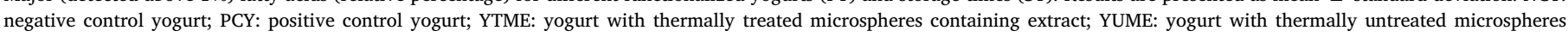
containing extract.

\begin{tabular}{|c|c|c|c|c|c|c|c|c|c|c|c|c|}
\hline & & $\mathrm{C} 6: 0$ & $\mathrm{C} 10: 0$ & $\mathrm{C} 12: 0$ & $\mathrm{C} 14: 0$ & $\mathrm{C} 16: 0$ & C18:0 & C18:1n9 & C18:2n6 & SFA & MUFA & PUFA \\
\hline \multirow[t]{5}{*}{ FY } & $\mathrm{NCY}$ & $3.0 \pm 0.3$ & $3.0 \pm 0.2$ & $3.4 \pm 0.2$ & $11.0 \pm 0.5$ & $29 \pm 1$ & $10.1 \pm 0.5$ & $25 \pm 1 \mathrm{a}$ & $2.7 \pm 0.4$ & $68 \pm 1 b$ & $27 \pm 1 \mathrm{a}$ & $4.6 \pm 0.5$ \\
\hline & PCY & $3.3 \pm 0.2$ & $3.1 \pm 0.2$ & $3.6 \pm 0.1$ & $11.1 \pm 0.4$ & $31 \pm 1$ & $10.0 \pm 0.5$ & $23 \pm 1 b$ & $2.5 \pm 0.1$ & $70 \pm 1 \mathrm{a}$ & $25 \pm 1 b$ & $4.3 \pm 0.1$ \\
\hline & YTME & $2.9 \pm 0.5$ & $3.1 \pm 0.2$ & $3.5 \pm 0.2$ & $11.1 \pm 0.4$ & $31 \pm 1$ & $9.8 \pm 0.4$ & $23 \pm 1 b$ & $2.5 \pm 0.2$ & $70 \pm 1 \mathrm{a}$ & $25 \pm 1 \mathrm{~b}$ & $4.4 \pm 0.2$ \\
\hline & YUME & $2.7 \pm 0.5$ & $3.2 \pm 0.2$ & $3.7 \pm 0.2$ & $11.5 \pm 0.4$ & $32 \pm 1$ & $9.9 \pm 0.5$ & $23 \pm 1 b$ & $2.6 \pm 0.3$ & $70 \pm 1 a$ & $25 \pm 1 b$ & $4.5 \pm 0.3$ \\
\hline & ANOVA $p$-value $(\mathrm{n}=18)^{2}$ & $<0.001$ & 0.016 & $<0.001$ & $<0.001$ & $<0.001$ & 0.265 & $<0.001$ & 0.110 & $<0.001$ & $<0.001$ & 0.129 \\
\hline \multirow[t]{3}{*}{ ST } & 0 days & $3.0 \pm 0.4$ & $3.1 \pm 0.2$ & $3.5 \pm 0.2$ & $11.1 \pm 0.4$ & $30 \pm 1$ & $9.9 \pm 0.5$ & $24 \pm 1$ & $2.8 \pm 0.2$ & $69 \pm 1$ & $26 \pm 1$ & $4.7 \pm 0.3$ \\
\hline & 7 days & $3.0 \pm 0.5$ & $3.1 \pm 0.2$ & $3.5 \pm 0.2$ & $11.2 \pm 0.5$ & $31 \pm 1$ & $10.0 \pm 0.5$ & $23 \pm 1$ & $2.4 \pm 0.1$ & $70 \pm 1$ & $26 \pm 1$ & $4.2 \pm 0.2$ \\
\hline & ANOVA $p$-value $(\mathrm{n}=36)^{3}$ & 0.444 & 0.751 & 0.585 & 0.132 & 0.031 & 0.274 & 0.019 & $<0.001$ & 0.002 & 0.058 & $<0.001$ \\
\hline $\mathrm{FY} \times \mathrm{ST}$ & $p$-value $(\mathrm{n}=72)^{4}$ & $<0.001$ & 0.020 & 0.010 & $<0.001$ & $<0.001$ & $<0.001$ & 0.573 & $<0.001$ & 0.210 & 0.342 & $<0.001$ \\
\hline
\end{tabular}

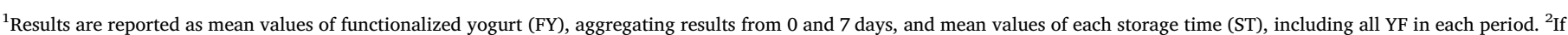

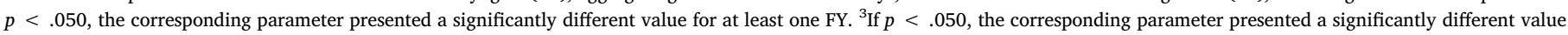
among both ST. ${ }^{4}$ If $p<.050$, the interaction among factors is significant; in this case, the multiple comparisons could not be classified.

function 3, forming a group in the negative side, in opposition to NCY and YTME, which were located in the positive side, mainly due to their differences on $\mathrm{C} 16: 0$ and $\mathrm{C} 18: 1 \mathrm{n} 9 \mathrm{c}$.

In the performed LDA, the classification performance was $100 \%$ accurate, either for original grouped cases as well as for the cross-validated grouped cases. Overall, it may be concluded that YTME represents the best yogurt formulations mainly due to its higher antioxidant activity. Some other specific differences were found among the remaining formulations, but their contribution to the global quality of yogurt is not as relevant as that provided by antioxidant activity. In addition, YTME was the only one able to increase the antioxidant activity along time, contrarily to the remaining formulations, which showed a general decrease in this type of bioactivity. Crosslinked citric acid- maltodextrin microspheres, particularly after being thermally treated, represent a feasible carrier to incorporate lipophilic bioactive agents in yogurt, as indicated by the results obtained with ergosterol.

\section{Conclusions}

The use of crosslinked citric acid-maltodextrin preparations as carrier material for the microencapsulation of Agaricus bisporus extracts is reported herein. The productive process was conducted by spray-drying technique which constitute a novelty in the present context. Thermal and spectroscopic characterization of the microspheres confirmed that maltodextrin reacted with citric acid leading to the desired crosslinked network. Also, it was possible to infer a slight positive effect of the thermal treatment on the crosslinking reaction extension. The extract was successfully incorporated in the microspheres and protected from degradation. Also, an increase in bioactivity was detected which may be associated with the possible esterification of the extract trough the reaction with citric acid. Additionally, the resulting products showed no toxicity to non-tumor cells.

The incorporation of Agaricus bisporus extract, in free and microencapsulated forms, in yogurts resulted in products with promising bioactive properties, with the sample TME (loaded-extract microspheres and subjected to post-thermal treatment) giving the best results in terms of bioactive properties preservation along storage. Results showed that the use of spray-drying technique and the occurrence of crosslinking of the encapsulating material are efficient to promote the protection, assuring a gradual release of the extract under the tested storage time.

\section{Acknowledgments}

This work was financially supported by: Project POCI-01-0145FEDER-006984 - Associate Laboratory LSRE-LCM funded by FEDER through COMPETE2020 - Programa Operacional Competitividade e Internacionalização (POCI) - and by national funds through FCT Fundação para a Ciência e a Tecnologia. The authors are also grateful to FCT and FEDER under Programme PT2020 for financial support to CIMO (UID/AGR/00690/2013), S. Heleno grant (SFRH/BPD/101413/ 2014) and L. Barros, J. Barreira and C. Calhelha contracts. To Norte 2020, through FEDER e FSE under PT2020 (Projeto NORTE-01-0145FEDER-000006). To FEEI through NORTE 2020 (Project ValorNatural $^{\circledR}$ ). To CAPES (Brasil) (Project 99999.000488/2016-00, Strategic programs -DRI).

\section{Appendix A. Supplementary data}

Supplementary data associated with this article can be found, in the 
online version, at http://dx.doi.org/10.1016/j.foodchem.2017.11.098.

\section{References}

Abreu, R. M. V., Ferreira, I. C. F. R., Calhelha, R. C., Lima, R. T., Vasconcelos, M. H. Adega, F., ... Queiroz, M. J. R. P. (2011). Anti-hepatocellular carcinoma activity using human HepG2 cells and hepatotoxicity of 6-substituted methyl 3-aminothieno[3,2-b] pyridine-2- carboxylate derivatives: In vitro evaluation, cell cycle analysis and QSAR studies. European Journal of Medicinal Chemistry, 46(12), 5800-5806. http://dx.doi. org/10.1016/j.ejmech.2011.09.029.

AOAC. (2016). Official methods of analysis of AOAC international. AOAC international, $20^{\mathrm{a}}$ ed.

Awadhiya, A., Kumar, D., \& Verma, V. (2016). Crosslinking of agarose bioplastic using citric acid. Carbohydrate Polymers, 151, 60-67. http://dx.doi.org/10.1016/j.carbpol. 2016.05.040.

Barreira, J. C. M., Oliveira, M. B. P. P., \& Ferreira, I. C. F. R. (2014). Development of a novel methodology for the analysis of ergosterol in mushrooms. Food Analytical Methods, 7(1), 217-223. http://dx.doi.org/10.1007/s12161-013-9621-9.

Barreira, J. C. M., Pereira, J. A., Oliveira, M. B. P. P., \& Ferreira, I. C. F. R. (2010). Sugars profiles of different chestnut (Castanea sativa Mill.) and almond (Prunus dulcis) cultivars by HPLC-RI. Plant Foods for Human Nutrition, 65(1), 38-43. http://dx.doi. org/10.1007/s11130-009-0147-7.

Breternitz, N. R., De V. Fidelis, C. H., Silva, V. M., Eberlin, M. N., \& Hubinger, M. D. (2017). Volatile composition and physicochemical characteristics of mussel (Perna perna) protein hydrolysate microencapsulated with maltodextrin and n-OSA modified starch. Food and Bioproducts Processing, 105, 12-25. http://dx.doi.org/10.1016/ j.fbp.2017.05.008.

Caleja, C., Barros, L., Antonio, A. L., Carocho, M., Oliveira, M. B. P. P., \& Ferreira, I. C. F. R. (2016). Fortification of yogurts with different antioxidant preservatives: A comparative study between natural and synthetic additives. Food Chemistry, 210, 262-268. http://dx.doi.org/10.1016/j.foodchem.2016.04.114.

Castro-Cabado, M., Parra-ruiz, F. J., Casado, A. L., \& Román, J. S. (2016). Thermal Crosslinking of Maltodextrin and Citric Acid. Methodology to Control the Polycondensation Reaction under Processing Conditions, 24(8), 643-654.

Corrêa, R. C. G., Peralta, R. M., Bracht, A., \& Ferreira, I. C. F. R. (2017). The emerging use of mycosterols in food industry along with the current trend of extended use of bioactive phytosterols. Trends in Food Science \& Technology, 67, 19-35. http://dx.doi. org/10.1016/j.tifs.2017.06.012.

Dias, M. I., Ferreira, I. C. F. R., \& Barreiro, M. F. (2015). Microencapsulation of bioactives for food applications. Food \& Function, 6(4), 1035-1052. http://dx.doi.org/10.1039/ C4FO01175A.

Gao, J. M., Wang, M., Liu, L. P., Wei, G. H., Zhang, A. L., Draghici, C., \& Konishi, Y. (2007). Ergosterol peroxides as phospholipase A2 inhibitors from the fungus Lactarius hatsudake. Phytomedicine, 14(12), 821-824. http://dx.doi.org/10.1016/j. phymed.2006.12.006.

Gaze, L. V., Oliveira, B. R., Ferrao, L. L., Granato, D., Cavalcanti, R. N., Conte Júnior, C. A., ... Freitas, M. Q. (2015). Preference mapping of dulce de leche commercialized in Brazilian markets. Journal of Dairy Science, 98(3), 1443-1454. http://dx.doi.org/10. 3168/jds.2014-8470.

Ghorbanzade, T., Jafari, S. M., Akhavan, S., \& Hadavi, R. (2017). Nano-encapsulation of fish oil in nano-liposomes and its application in fortification of yogurt. Food Chemistry, 216, 146-152. http://dx.doi.org/10.1016/j.foodchem.2016.08.022.

Gil-Ramírez, A., Ruiz-Rodríguez, A., Marín, F. R., Reglero, G., \& Soler-Rivas, C. (2014). Effect of ergosterol-enriched extracts obtained from Agaricus bisporus on cholesterol absorption using an in vitro digestion model. Journal of Functional Foods, 11(C), 589-597. http://dx.doi.org/10.1016/j.jff.2014.08.025.

Granato, D., Nunes, D. S., \& Barba, F. J. (2017). An integrated strategy between food chemistry, biology, nutrition, pharmacology, and statistics in the development of functional foods: A proposal. Trends in Food Science and Technology, 62, 13-22. http://dx.doi.org/10.1016/j.tifs.2016.12.010 (December 2016).

Heleno, S. A., Barros, L., Martins, A., Queiroz, M. J. R. P., Santos-Buelga, C., \& Ferreira, I. C. F. R. (2012). Phenolic, polysaccharidic, and lipidic fractions of mushrooms from northeastern portugal: Chemical compounds with antioxidant properties. Journal of Agricultural and Food Chemistry, 60(18), 4634-4640. http://dx.doi.org/10.1021/ jf300739m.

Heleno, S. A., Barros, L., Sousa, M. J., Martins, A., \& Ferreira, I. C. F. R. (2009). Study and characterization of selected nutrients in wild mushrooms from Portugal by gas chromatography and high performance liquid chromatography. Microchemical Journal, 93(2), 195-199. http://dx.doi.org/10.1016/j.microc.2009.07.002.

Heleno, S. A., Diz, P., Prieto, M. A., Barros, L., Rodrigues, A., Barreiro, M. F., \& Ferreira, I. C. F. R. (2016). Optimization of ultrasound-assisted extraction to obtain mycosterols from Agaricus bisporus L. by response surface methodology and comparison with conventional Soxhlet extraction. Food Chemistry, 197, 1054-1063. http://dx.doi.org/ 10.1016/j.foodchem.2015.11.108.

Heleno, S. A., Rudke, A. R., Calhelha, R. C., Carocho, M., Barros, L., Gonçalves, O. H., ... Ferreira, I. C. F. R. (2017). Development of dairy beverages functionalized with pure ergosterol and mycosterol extracts: An alternative to phytosterol-based beverages. Food \& Function, 8(1), 103-110. http://dx.doi.org/10.1039/C6FO01600F.

Janiaski, D. R., Pimentel, T. C., Cruz, A. G., \& Prudencio, S. H. (2016). Strawberry-flavored yogurts and whey beverages: What is the sensory profile of the ideal product? Journal of Dairy Science, 99(7), 5273-5283. http://dx.doi.org/10.3168/jds.201510097.

Karaaslan, M., Ozden, M., Vardin, H., \& Turkoglu, H. (2011). Phenolic fortification of yogurt using grape and callus extracts. LWT - Food Science and Technology, 44(4), 1065-1072. http://dx.doi.org/10.1016/j.lwt.2010.12.009.

Karam, M. C., Gaiani, C., Hosri, C., Burgain, J., \& Scher, J. (2013). Effect of dairy powders fortification on yogurt textural and sensorial properties: A review. Journal of Dairy Research, 80(4), 400-409. http://dx.doi.org/10.1017/S0022029913000514.

Kaur, N., \& Singh, D. P. (2017). Deciphering the consumer behaviour facets of functional foods: A literature review. Appetite, 112, 167-187. http://dx.doi.org/10.1016/j. appet.2017.01.033.

Khandal, D., Aggarwal, M., Suri, G., \& Coqueret, X. (2015). Electron beam irradiation of maltodextrin and cinnamyl alcohol mixtures: Influence of glycerol on cross-linking. Carbohydrate Polymers, 117, 150-159. http://dx.doi.org/10.1016/j.carbpol.2014.08. 115.

Menzel, C., Olsson, E., Plivelic, T. S., Andersson, R., Johansson, C., Kuktaite, R., ... Koch, K. (2013). Molecular structure of citric acid cross-linked starch films. Carbohydrate Polymers, 96(1), 270-276. http://dx.doi.org/10.1016/j.carbpol.2013.03.044.

Moser, P., Telis, V. R. N., de Andrade Neves, N., García-Romero, E., Gómez-Alonso, S., \& Hermosín-Gutiérrez, I. (2017). Storage stability of phenolic compounds in powdered BRS Violeta grape juice microencapsulated with protein and maltodextrin blends. Food Chemistry, 214, 308-318. http://dx.doi.org/10.1016/j.foodchem.2016.07.081.

Nawong, S., Oonsivilai, R., Boonkerd, N., \& Truelstrup Hansen, L. (2016). Entrapment in food-grade transglutaminase cross-linked gelatin-maltodextrin microspheres protects Lactobacillus spp. during exposure to simulated gastro-intestinal juices. Food Research International, 85, 191-199. http://dx.doi.org/10.1016/j.foodres.2016.04.041.

Pereira, E., Barros, L., Barreira, J. C. M., Carvalho, A. M., Antonio, A. L., \& Ferreira, I. C. F. R. (2016). Electron beam and gamma irradiation as feasible conservation technologies for wild Arenaria montana L.: Effects on chemical and antioxidant parameters. Innovative Food Science \& Emerging Technologies, 36, 269-276. http://dx.doi.org/10. 1016/j.ifset.2016.07.012.

Pereira, E. P. R., Faria, J. A. F., Cavalcanti, R. N., Garcia, R. K. A., Silva, R., Esmerino, E. A., ... Cruz, A. G. (2016). Oxidative stress in probiotic Petit Suisse: Is the jabuticaba skin extract a potential option? Food Research International, 81, 149-156. http://dx doi.org/10.1016/j.foodres.2015.12.034.

Reis, F. S., Heleno, S. A., Barros, L., Sousa, M. J., Martins, A., Santos-Buelga, C., \& Ferreira, I. C. F. R. (2011). Toward the antioxidant and chemical characterization of mycorrhizal mushrooms from Northeast Portugal. Journal of Food Science, 76(6), 824-830. http://dx.doi.org/10.1111/j.1750-3841.2011.02251.x.

Reis, F. S., Martins, A., Vasconcelos, M. H., Morales, P., \& Ferreira, I. C. F. R. (2017). Functional foods based on extracts or compounds derived from mushrooms. Trends in Food Science \& Technology, 66, 48-62. http://dx.doi.org/10.1016/j.tifs.2017.05.010.

Ribeiro, A., Ruphuy, G., Lopes, J. C., Dias, M. M., Barros, L., Barreiro, F., \& Ferreira, I. C. F. R. (2015). Spray-drying microencapsulation of synergistic antioxidant mushroom extracts and their use as functional food ingredients. Food Chemistry, 188, 612-618. http://dx.doi.org/10.1016/j.foodchem.2015.05.061.

Santillán-Urquiza, E., Méndez-Rojas, M.Á., \& Vélez-Ruiz, J. F. (2017). Fortification of yogurt with nano and micro sized calcium, iron and zinc, effect on the physicochemical and rheological properties. LWT - Food Science and Technology, 80, 462-469. http://dx.doi.org/10.1016/j.lwt.2017.03.025.

Shao, S., Hernandez, M., Kramer, J. K. G., Rinker, D. L., \& Tsao, R. (2010). Ergosterol profiles, fatty acid composition, and antioxidant activities of button mushrooms as affected by tissue part and developmental stage. Journal of Agricultural and Food Chemistry, 58(22), 11616-11625. http://dx.doi.org/10.1021/jf102285b.

Singh, G., \& Muthukumarappan, K. (2008). Influence of calcium fortification on sensory, physical and rheological characteristics of fruit yogurt. LWT - Food Science and Technology, 41(7), 1145-1152. http://dx.doi.org/10.1016/j.lwt.2007.08.027.

Stone, S. A., Gosavi, P., Athauda, T. J., \& Ozer, R. R. (2013). In situ citric acid crosslinking of alginate/polyvinyl alcohol electrospun nanofibers. Materials Letters, 112, 32-35. http://dx.doi.org/10.1016/j.matlet.2013.08.100.

Taofiq, O., Calhelha, R. C., Heleno, S., Barros, L., Martins, A., Santos-Buelga, C., ... Ferreira, I. C. F. R. (2015). The contribution of phenolic acids to the anti-inflammatory activity of mushrooms: Screening in phenolic extracts, individual parent molecules and synthesized glucuronated and methylated derivatives. Food Research International, 76, 821-827. http://dx.doi.org/10.1016/j.foodres.2015.07.044.

Wang, L., Zheng, M., Wang, Y., Zhang, Y., Qian, H., Zhang, H., \& Qi, X. (2014). Antidiabetic activity of cassava cross-linked octenyl succinic maltodextrin in STZ-induced diabetic mice. International Journal of Biological Macromolecules, 64, 247-251. http:// dx.doi.org/10.1016/j.ijbiomac.2013.11.017.

Yasukawa, K., Aoki, T., Takido, M., Ikekawa, T., Saito, H., \& Matsuzawa, T. (1994). Inhibitory effects of ergosterol isolated from the edible mushroom Hypsizigus marmoreus on TPA-induced inflammatory ear oedema and tumour promotion in mice. Phytother Research, 8(1), 10-13. http://dx.doi.org/10.1002/ptr.2650080103. 